\section{Oral Manifestations of Inherited Disorders}

By Heddie O. Sedano, John J. Sauk, and Robert J. Gorlin. (Pp. viii + 214; Figures + Tables. US \$48.95; Dfl.120; £14.50.) Sevenoaks: Butterworth. 1977.

This book is a compendium of the many inherited disorders that have characteristic oral and facial manifestations. Each condition is described briefly under the headings of facies, oral structures, systemic features, differential diagnosis, and laboratory aids. Synonyms are listed and some references are given. It is impossible to devise a logical system of classification for conditions of such diversity, and this text is ordered according to the orodental structures primarily affected. This has the advantage that the clinician will often be able to identify an unfamiliar condition after minimal searching. However, the converse does not apply because the index is quite inadequate: neither the preferred name nor synonyms are included. Thus the clinician ignorant of the facial manifestations of Crouzon's syndrome, for example, would have to search the entire volume. If he were fortunate, he might spot Crouzon's syndrome mentioned as a synonym for craniofacial dysostosis.

This book may be useful for medical and dental practitioners who wish to consult a concise volume of this type. However, for most purposes, the now classic Syndromes of the Head and Neck by R. J. Gorlin, J. J. Pindborg, and M. M. Cohen will remain the text of choice because of its wider scope, its better illustrations, and its superior index. In fact, the volume under review is merely an abstract of the larger book, the descriptions being paraphrased from the latter and many illustrations being common to both. On the subject of illustrations, it is unfortunate that so many of the intraoral photographs and $x$-rays have been inverted. It may be difficult for the printer to tell which way up the teeth should be, but the 3 authors are dentally qualified.

In summary, this is a reliable text of reference, though the lack of an adequate index is a serious deficiency. The organisation of the book is sensible, but still does not provide the complete answer for the clinician who wishes to identify a syndrome without plodding through an entire reference work page by page. Whether there is really a place for another volume in a field which is already so well covered is open to question.

W. J. B. Houston

\section{Oral Facial Genetics}

Edited by Ray E. Stewart and Gerald H. Prescott. (Pp. xii + 680; Figures + Tables. £38.60.) St. Louis: C. V. Mosby; London: Henry Kimpton. 1976.

This text, edited by Ray Stewart and Gerald Prescott, helps to bridge a gap between existing books on medical genetics and those devoted to orofacial anomaly. The editors are assisted by 15 distinguished colleagues from a number of North American institutions, each contributing to 1 or more chapters in their field of special interest.The first 3 chapters are devoted to the general principles of genetics and the genetic mechanisms of control during early orofacial development and craniofacial morphogenesis.

Following these are 3 chapters dealing with the genetic background to the 2 common dental diseases of dental caries and periodontal disease, and the genetic factors influencing the development of malocclusions, tooth size, and anomalous tooth development.

Four separate chapters are given to a detailed consideration of the inherited disorders of enamel, dentine, cementum and periodontal tissues, and the dental pulp. Apart from 1 isolated chapter dealing with the genetics of cleft lip and palate, and a brief mention of transplantation genetics, the remainder of the book is given over to a consideration of the orofacial manifestations associated with immunological disorders, inherited mucocutaneous disorders, inborn errors of metabolism, haematological abnormalities, cytogenic anomalies, and dysmorphic syndromes.

The book is well written, easy to read, and beautifully illustrated by many fine photographs and line drawings. For each of the subjects covered there is a comprehensive and reasonably up-to-date bibliography.

A problem common to many texts employing multiple authors is noticeable in this volume, namely that there is occasional repetition of material. Provided that the contributors agree, this does not present too much difficulty; disagreement, however, may lead to confusion. An example of this is seen in chapters 7 and 8 , where different dental manifestations are claimed for the hemizygous male affected by pseudohypoparathyroidism. Another slight irritation is the tendency to pad out chapters with material the relevance of which is difficult to establish. This is present in chapter 9 where taurodontism, an anomaly of tooth 
form, is discussed under the heading of primary diseases of cementum. These are, however, small criticisms of a generally excellent text which should be of considerable value to clinicians in many branches of medicine and dentistry.

\section{G. B. WINTER}

\section{The Eye in Chromosome Duplications and Deficiencies}

By Marcelle Jay. (Pp. xi + 249; Figures + Tables. SFr4.74.) Basel and New York: Marcel Dekker. 1977.

Ophthalmologists have long been interested in genetics. One of the first textbooks on genetics was written in 1934 by an ophthalmologist, Waardenburg, and the tradition has been carried on by Franceschetti and, in this country, by Sorsby. Some ophthalmic hospitals have a genetic clinic (the author helps her husband at the one at Moorfields, London), so that this book, though not written by a clinician, has its origins in the clinic.

Since the advent of banding techniques, many new chromosomal syndromes have been described. Clinicians are aware that eye anomalies appear in many different syndromes, so that the presence of hypertelorism, ptosis, strabismus, or epicanthus are not helpful diagnostic criteria. If less nonspecific features, such as optic atrophy or colobomata, were to be found in certain of the aneuploidies, these would be more useful clinical signs. The main purpose of this book is to describe and tabulate the clinical findings, with particular emphasis on the ocular anomalies in the partial deletions and trisomies. As the book has its origin in a thesis, the detail is excellent and there is a comprehensive coverage of references since the introduction of banding in 1972. In addition, there is a concise first chapter on the chromosome and a useful glossary of terms. The interesting association of retinoblastoma with partial deletion of chromosome 13 is also dealt with. Perhaps potential readers should be warned that duplications and deletions referred to in the title do not include whole chromosomes, so that trisomy 13, trisomy 18, and even Down's syndrome are not described. Surprisingly, an exception is made for Turner's syndrome. Nevertheless, this is a most useful reference work for those dealing with the 'funny looking kid' syndromes.

M. BARAITSER

\section{Myotonia Congenita and Syndromes Associated with Myotonia. Clinical-Genetic Studies of the Nondystro- phic Myotonias}

By Peter Emil Becker, with contributions by Rainer Knussmann and Erick Kuhn. Translated by Mary F. Passarge. (Pp. $x+181 ; 146$ Figures +40 Tables. DM.78.) Stuttgart: Georg Thieme. 1977.
Myotonia refers to the phenomenon of delayed relaxa- $\Omega$ tion after either voluntary contraction or contraction $\stackrel{\mathbb{}}{?}$ induced by percussion. The first clear and thorough $\stackrel{\mathbb{P}}{+}$ description of a dominantly inherited form of myo- $\overrightarrow{\vec{F}}$ tonia was by $\mathrm{Dr}$ Asmus Julius Thomsen 100 years ago, who observed the disease in himself as well as in other members of his family. Since then it has become $\frac{\bar{c}}{\bar{c}}$. increasingly clear that the inherited myotonic syn- $\frac{\vec{\sigma}}{\sigma}$ dromes are a very heterogeneous group of disorders. $\unrhd$ In fact some 15 clinically distinct forms have now been $\%$ described. Professor Becker has been particularly $\vec{O}$ interested in these disorders, and this detailed monograph concerns his studies of 142 probands with $\vec{\omega}$ established myotonia congenita and their relatives, including Thomsen's original family. His major $\overline{3}$ contribution has been to show the clear distinction between dominant and recessive forms of myotonia $\rightarrow$ congenita. The former tend to have an earlier onset, to be more variable, less severe, and less often associ- ${ }_{-}$ ated with persistent weakness than the latter. There appear to be at least 3 clinically distinct forms of 운 dominant myotonia congenita: Thomsen's disease itself, a form associated with myalgia, and another $?$ with marked cold dependence.

Becker observed the prevalence of dominant $\stackrel{\text { क }}{\longrightarrow}$ myotonia congenita in West Germany to be about 1 in $\vec{\omega}$ 500000 , though this is clearly an underestimate for $\infty$ often symptoms are mild and ascertainment therefore not complete. In fact, he thinks the true prevalence may be as high as 1 in 23000 , about twice the prevalence of recessive myotonia congenita. Interestingly, $\overline{\mathrm{D}}$ malignant hyperthermia was not observed in any of $\frac{\varrho}{\varnothing}$ the families he studied.

Besides presenting detailed clinical findings, the $\overrightarrow{\vec{O}}$ monograph concludes with 2 brief sections on bio- 3 chemistry (by Kuhn) and multivariate analysis (by Knussmann). The results of the biochemical studies? (serum and muscle enzymes, serum electrolytes, serum and muscle lipids) are unrevealing, apart from $\bullet$ abnormalities in the lipid composition of muscle in dominant myotonia congenita, the relevance of which is not yet clear. With regard to the statistical analyses, $\delta$ an attempt was made to verify Becker's classification firstly by testing the significance between the 웅 myotonic types he described, and secondly by $D$ attempting to establish an independent classification from the original data. In general, both approaches $\bar{N}$ tended to confirm Becker's intuitive classification.

Altogether this is a very valuable monograph and ${ }^{N}$ can be unreservedly recommended to anyone with an interest in this complex group of disorders. It also provides an excellent model for anyone embarking on $a_{0}$ clinical-genetic study. It is gratifying to those with $\underset{\mathbb{D}}{\overparen{D}}$ primarily clinical interests that this approach is still $\stackrel{\oplus}{\stackrel{\oplus}{+}}$ very worth while and can produce valuable results as 7 well as being intellectually satisfying.

Alan E. H. EMERY 